\title{
Epidural Migration of Dervan Platelet Fibrin Plug Causing Recurrent Sciatica: A Case Report
}

\author{
Kanthila Mahesha \\ Department of Orthopaedics, Maithri Speciality Clinics Bendoorwell, Mangalore, Karnataka State India
}

Corresponding Author:

Kanthila Mahesha, MD, MS, DNB

Department of Orthopaedics, Maithri

Speciality Clinics Bendoorwell, Mangalore

575002, Karnataka state India

Tel: +91-824-2446068

Fax: +91-824-2218483

E-mail:maheshakb@yahoo.com

Received: April 30, 2020

Revised: July 11, 2020

Accepted: July 15, 2020
A 40-year-old male presented with right sided sciatica due to L3-L4 disc prolapse. Patient was treated with transforaminal endoscopic discectomy L3-L4 and platelet fibrin plug application under local anaesthesia. Patient had good relief from pain after the procedure. At one month after the surgery, patient developed recurrent sciatica. Magnetic resonance imaging revealed recurrent compression at the operated level. Patient was treated with repeat transforaminal endoscopic surgery. Transforaminal endoscopy revealed epidural migration of the platelet fibrin plug and was removed. Patients had immediate relief from radicular pain with no further recurrence. Histopathology report confirmed that it was migrated platelet fibrin plug. As the platelet fibrin plug does not have any fixation in the disc, it carries a significant risk of epidural migration and recurrent sciatica.

Key Words: Disc prolapse, Platelet rich fibrin, Transforaminal endoscopy

\section{INTRODUCTION}

Percutaneous endoscopic discectomy is a safe and effective technique for lumbar disc prolapse ${ }^{1)}$. Recurrent disc prolapse is a major concern after lumbar disc surgery. Annular defect of $6 \mathrm{~mm}$ or more is associated with increased risk of recurrent disc prolapse ${ }^{2)}$. Intervertebral disc engineering strategies are increasingly focusing on the regeneration or repair of the annulus fibrosus in order to reduce the number of re-herniations ${ }^{3)}$. There are mechanical and biological solutions for annular defects. One of the suggested biological solutions for annular healing is platelet fibrin plug $^{4)}$. Several in vitro studies showed that platelet-rich preparations increased the matrix production and cell number and may therefore be considered to promote annulus fibrosus repair ${ }^{5}$. The idea to use platelet fibrin plug to promote annulus fibrosus repair had been tested recently. We report a patient who had recurrent sciatica caused by epidural migration of platelet fibrin which was applied after percutaneous transforaminal endoscopic discectomy. In our best knowledge, this complication is reported for the first time in the literature.

\section{CASE REPORT}

A 40 years old male patient presented with right sided sciatica which started 6 months ago. Patient had taken conservative treatment without any relief. On examination, straight leg raising test was positive at 45 degrees on right side. Femoral stretch test was positive on right side. There was no motor or sensory deficit. His right knee jerk was decreased. Magnetic resonance
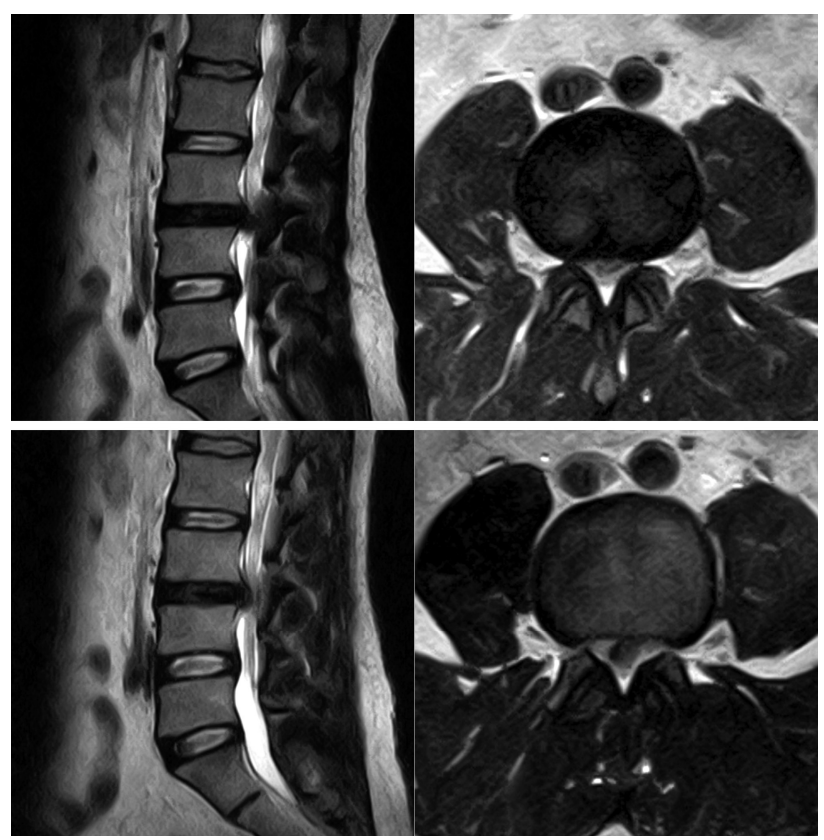

Fig. 1. Pre-operative MRI scan showing right paracentral disc extrusion at L3-L4 level. 
imaging (MRI) scan lumbosacral spine showed right paracentral disc extrusion at L3-L4 level causing significant compression of thecal sac and nerve roots (Fig. 1). We performed a right transforaminal endoscopic discectomy at L3-4 under local anaesthesia. An extruded disc fragment sized of $2 \times 0.6 \mathrm{~cm}$ was removed. After adequate decompression of the dural sac, the annular defect was measured about $0.5 \times 1.5 \mathrm{~cm}$. A platelet fibrin plug was prepared as described in the previous studies ${ }^{6,7)}$. In brief, $10 \mathrm{~mL}$ of patient's peripheral blood was obtained, and centrifuged aseptically at 3,000 rpm for 10 minutes. After a resting period of 10 minutes, platelet fibrin plug is removed

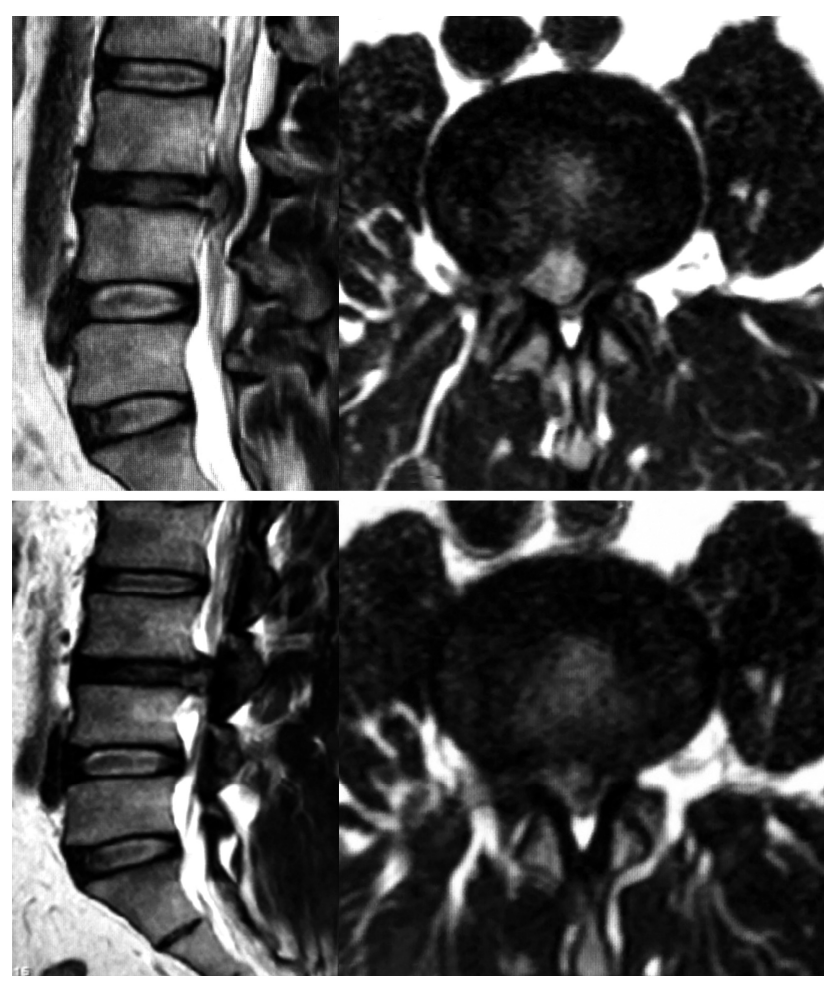

Fig. 2. Repeat MRI scan showing midline protrusion at $L 3-L 4$ level causing severe compression of thecal sac and nerve roots.

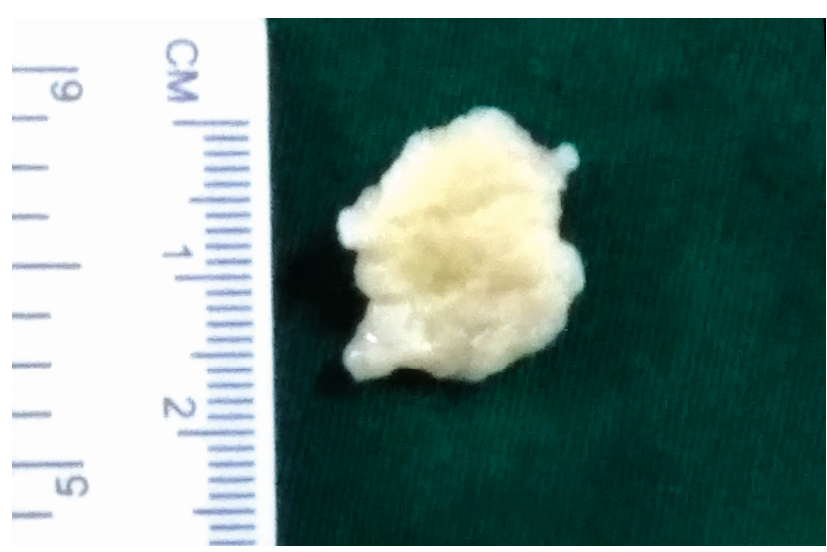

Fig. 3. Platelet fibrin plug removed by transforaminal surgery. aseptically, washed with saline and cut to desired size depending on annular defect. There was no intra operative complication, and the patient's leg pain was completely resolved postoperatively. He was discharged in a day. A month after the initial surgery, the patient's symptom recurred. The pain became progressively severe, and did not improve with conservative treatment. On examination, no neurologic deficit was observed. However, right straight leg raising test was limited to 45 degrees, and femoral stretch test was positive on right side. Follow-up MRI scan showed a posterior midline protrusion at L3-4 level causing severe canal stenosis and compression of the thecal sac and nerve roots, suggesting recurrent disc herniation (Fig. 2). The patient underwent another transforaminal endoscopic surgery. Under the endoscopic view, we discovered a distinct pale yellow coloured tissue was compressing the thecal sac. It was removed in one piece. It was avascular, firm in consistency, and measured about $1.8 \times 1.2 \mathrm{~cm}$ (Fig. 3). The dural sac was highly inflamed. Disc looked normal without any sign of inflammation. Post-operative MRI showed good decompression of the thecal sac and nerve roots (Fig. 4). Histopathology revealed large clumps of degenerated platelets surrounded by fibrinoid material with focal hyalinization (Fig. 5). After the surgery, the leg pain disappeared, and patient was discharged in a day. At 2 years of follow-up, the patient was symptom free.

\section{DISCUSSION}

The limited intrinsic healing capacity of the annulus fibrosus negatively affects the success rates of discectomies ${ }^{3)}$. There

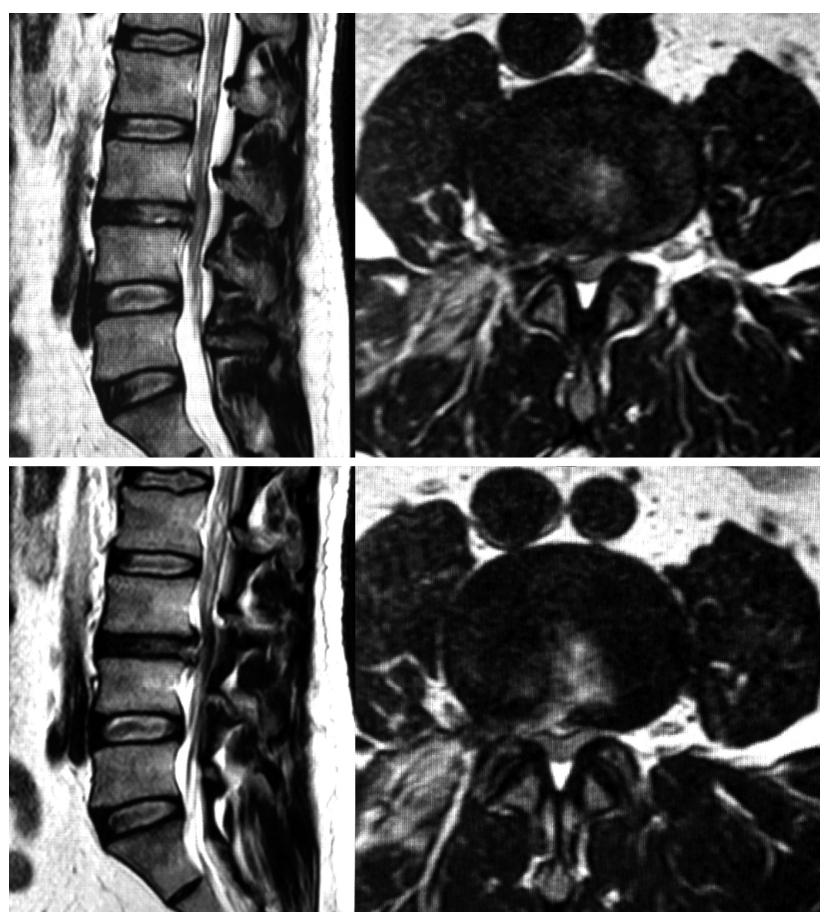

Fig. 4. Post-operative MRI scan showing good decompression at L3-L4 level. 


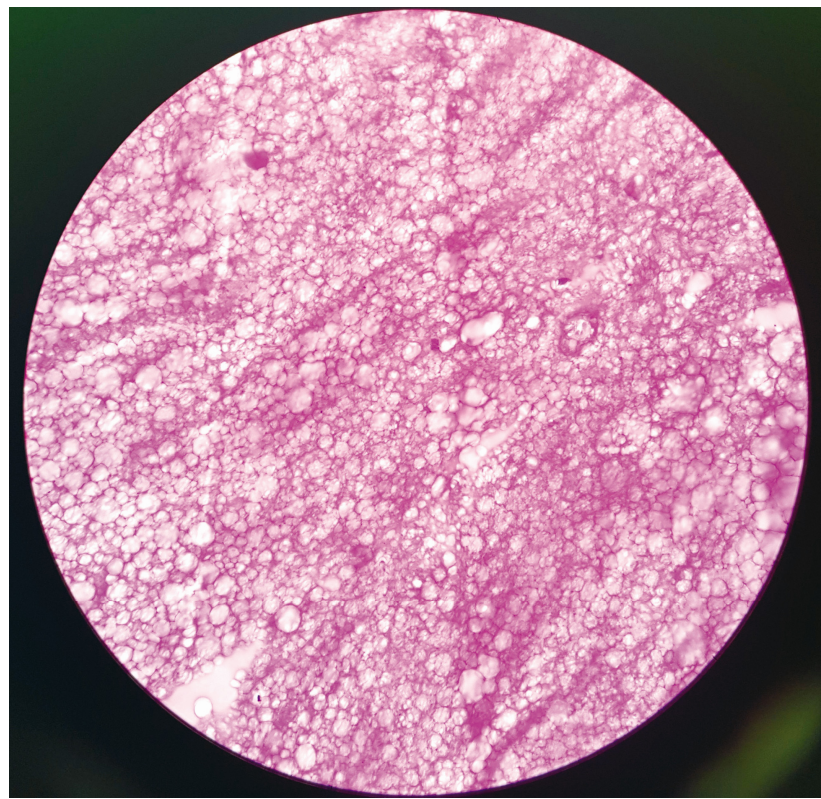

Fig. 5. Histopathology picture of migrated platelet fibrin plug.

are annular closure techniques and regenerative strategies to deal with damaged annulus fibrosus ${ }^{3)}$. Annular closure techniques are primarily focussing on restoration of the mechanical integrity of the annulus fibrosus and do offer clear solutions for delivery and fixation. Regenerative therapies, on the other hand, target the engineering of healthy and functional annulus fibrosus tissue ${ }^{3)}$. However, they lack implantation and fixation strategies that their clinical applications are limited.

Kienzler et al. reported that addition of a bone-anchored annular closure device (ACD) to lumbar discectomy in patients with large post-surgical annular defects reduces the risk of reherniation and reoperation ${ }^{8)}$. However, it requires more invasive surgery for implantation with added cost of implant. ACD was associated with distinct risks such as implantation difficulties, device migration, mesh detachment, and vertebral endplate changes ${ }^{8}$. There is no long term data available about ACD. Gelstix ${ }^{\mathrm{TM}}$ is a hydrogel implant indicated in discogenic back pain when there is no annular tear. Durdag et al. reported epidural fragmentation of hydrogel implant causing sciatica which required surgery".

Intradiscal platelet-rich plasma (PRP) is a safe and a possibly effective treatment for discogenic low back pain ${ }^{10)}$. Activated PRP works as a concentrate of multiple growth factors such as insulin-like growth factor-1 (IGF-1), platelet-derived growth factor (PDGF), transforming growth factor- $\beta$ (TGF-b), and basic fibroblast growth factor (bFGF) ${ }^{11)}$. These growth factors promote wound healing. Nadkarni et al. reported that use of platelet fibrin plug reduces post-operative pain, spasm and discomfort ${ }^{4}$. However, the prospect of this PRP strategy seems grim. Intervertebral disc represents a very complex joint in terms of cellular, biochemical, and mechanical elements. It is the largest acellular and avascular organ ${ }^{111}$. The avascular nature of the intervertebral disc, poor nutrition and severe biomechanical demands placed on it are all refractory to repair processes and it consequently has an intrinsically poor healing capability ${ }^{12}$. This complex harsh environment is the reason for failure of regenerative therapies ${ }^{111}$. Although these growth factors promote wound healing, it is not possible for the platelet rich fibrin plug to regenerate the annulus.

In the present case, platelet fibrin plug remained in the disc space as a solid mass for one month and then it migrated to the epidural space causing compression of thecal sac and nerve roots causing recurrent sciatica. This compression by the platelet fibrin plug was more than the initial compression by the disc. On the MRI, platelet fibrin plug appeared hyperintense on T2 weighted images and hypointense on $\mathrm{T} 1$ weighted images. It is difficult to differentiate between a nuclear herniation and migrated platelet plug on MRI. A soft recurrent disc herniation may resolve with conservative treatment, but a migrated platelet fibrin plug is unlikely to resolve. Patient required second surgery to remove the platelet fibrin plug due to severe pain. During the second surgery, there was no disc herniation. Migration of the platelet fibrin plug was not due to recurrent disc prolapse. Recurrent sciatica was entirely due to the migrated platelet fibrin plug.

The exact cause for migration of the platelet plug is not known. One of the reason for migration is lac of fixation in the disc space. With time, the water content in the plug reduces and plug shrinks in size. Gelatinous platelet fibrin plug becomes firm and more solid over a period of time. Any increase in intradiscal pressure can push the plug into the epidural space. There was no inflammation of the plug because of autologous origin. The inflammation was seen only at the dural surface due to mechanical compression. Once the plug is inserted into the disc space, it remains as a solid mass at least for few months. As the disc space is avascular, there is no way body can dissolve it easily. Histopathology report of the platelet fibrin plug showed large clumps of degenerated platelets surrounded by fibrinoid material with focal hyalinization. These histopathology findings question the possibility of biologic repair by the platelet fibrin plug. There are no long term studies available about the fate of platelet fibrin plug. Apart from questionable regenerative capability of the plug, risk of infection is also a concern for applying platelet fibrin plug after discectomy. This was the tenth case where the author had used platelet fibrin plug. After this complication, author has stopped using the platelet fibrin plug. At present, anuular defects are treated with radiofrequency ablation at the end of endoscopic disc surgery. The issue of annular defect will continue to haunt surgeons and patients for many years.

\section{CONCLUSION}

Platelet fibrin plug may have some benefits with regard to back pain in the immediate post-operative period, but there is a significant risk of epidural migration causing mechanical compression of the neural structures and recurrent sciatica. 
Conflicting Interest: Author has no conflict of interest.

\section{REFERENCES}

1. Mahesha K: Percutaneous endoscopic lumbar discectomy: Results of first 100 cases. Indian J Orthop 51(1):36-42, 2017

2. Carragee EJ, Spinnickie AO, Alamin TF, Paragioudakis S: A prospective controlled study of limited versus subtotal posterior discectomy: Short-term outcomes in patients with herniated lumbar intervertebral discs and large posterior annular defect. Spine (Phila Pa 1976) 31:653-657, 2006

3. Bron JL, Helder MN, Meisel HJ, Barend J, Royen V, Smit TH: Repair, regenerative and supportive therapies of the annulus fibrosus: Achievements and challenges. Eur Spine J 18:301-313, 2009

4. Nadkarni S, Kohli P, Gore S, Patel B: Use of biological solutions for annular healing: Dervan platelet fibrin plug in transforaminal disc surgery. IOSR-JDMS 15:52-57, 2016

5. Pirvu TN, Schroeder JE, Peroglio M, Verrier S, Kaplan L, Richardset RG, et al: Platelet-rich plasma induces annulus fibrosus cell proliferation and matrix production. Eur Spine J 23(4):745-753, 2014

6. Dohan DM, Choukroun J, Diss A, Dohan SL, Dohan AJJ,
Mouhyi J, et al: Platelet-rich fibrin (PRF): A second-generation platelet concentrate. Part II: Platelet-related biologic features. Oral Surg Oral Med Oral Pathol Oral Radiol Endod 101:E4550, 2006

7. Raaj V, Gautam A, Abhishek, Kumari P: Platelet-rich fibrin (PRF): A new generation platelet concentrate. Int J Dent Med Res 1(6): 164-167, 2015

8. Kienzler JC, Klassen PD, Miller LE, Assaker R, Heidecke V, Fröhlich S: Three-year results from a randomized trial of lumbar discectomy with annulus fibrosus occlusion in patients at high risk for reherniation. Acta Neurochir 161:1389-1396, 2019

9. Durdag E, Ayden O, Albayrak S, Atci IB, Armagan E: Fragmentation to epidural space: First documented complication of Gelstix (TM). Turk Neurosurg 24(4):602-605, 2014

10. MonfettM, Harrison J, Boachie-Adjei K, Lutz G: Intradiscal platelet-rich plasma (PRP) injections for discogenic low back pain: An update. Int Orthop 40(6):1321-1328, 2016

11. Buser Z., Chung A.S, Abedi A, Wang J: The future of disc surgery and regeneration. Int Orthop 43:995-1002, 2019

12. Melrose J, Smith SM, Little CB, Moore RJ, Roberts BV, Fraser $\mathrm{RD}$ : Recent advances in annular pathobiology provide insights into rim-lesion mediated intervertebral disc degeneration and potential new approaches to annular repair strategies. Eur Spine J 17(9):1131-1148, 2008 